Vol. 18 (2009): 103-116.

\title{
Exploring the physiological basis of cryopreservation success and failure in clonally propagated in vitro crop plant germplasm
}

\author{
Keith Harding ${ }^{1}$, Jason W. Johnston ${ }^{2}$ and Erica E Benson ${ }^{1}$ \\ ${ }^{1}$ Damar Research Scientists, Conservation, Environmental Science \& Biotechnology, Damar, Drum Road, \\ Cuparmuir, Fife, KY15 5RJ, Scotland, UK \\ ${ }^{2}$ HortResearch, 120 Mt Albert Road, Private Bag 92 169, Mt Albert, Auckland, New Zealand \\ e-mail:k.harding-damar@tiscali.co.uk
}

\begin{abstract}
An appraisal of potato and Ribes shoot meristem cryopreservation shows physiological factors influence survival and development, sometimes independently of protocol and genotype. Markers for oxidative damage incurred by cryostorage reveal two responses: (1) oxidative stress with an eventual decline in regrowth and (2) an oxidative burst associated with higher survival. Differential responses to cryoinjury are discussed in relation to in vitro ageing and genetic stability within the conceptual framework of cryobionomics. The possibility that cryopreservation-induced cell death and apoptosis occurs in plants is considered with respect to current concepts of animal cell cryoinjury. It is proposed that a more holistic approach is now required to understand the basis for success or failure of cryopreserved plant germplasm.
\end{abstract}

Key-words: apoptosis, ageing, clonal, cryopreservation, physiology, potato, Ribes, stability

\section{Introduction}

Cryostorage success cannot always be attributed to genotypic or cryogenic factors (cryoprotection, cooling and freezing) therefore, it is vital to understand the role of decisive physiological factors in cryopreservation failure. Several reasons may account for this: (1) restricted application to highly intolerant genotypes within a crop; (2) variable responses within and across genotypes; (3) low number of samples surviving cryostorage; (4) inability of meristems to regrow shoots and (5) idiosyncratic variability within and between experiments. While 
Harding, K. Exploring cryopreservation in clonally propagated crops

an acceptable number of germplasm accessions held in working cryobanks respond satisfactorily to cryopreservation, the application of otherwise successful protocols to some genotypes results in low survival (Keller et al. 2008, Panis et al. 2005). This has proven to be a persistent problem across different species. To address these issues, we examine the responses of clonal crop germplasm within the remit of the European CRYOPLANET - COST Action 871 project (Panis 2007). The effects of cryogenic and non-cryogenic factors are considered specifically for the recovery of potato and Ribes, as they provide exemplars of tuber and woody perennial crops. Shoot tip meristem responses to cryopreservation are evaluated using a reappraisal of the authors' earlier potato literature in the context of new studies on Ribes.

\section{Materials and Methods}

In vitro shoot cultures of Solanum tuberosum cultivars (cvs) 'Golden Wonder' and 'Desiree' were established on Murashige and Skoog (MS) medium (Benson et al. 1989). The effects of pre- and postcryopreservation light (high/low irradiance) treatments were studied on cryoprotected meristems, which were cryopreserved by ultra-rapid freezing (Benson et al. 1989) or controlled rate cooling (Harding et al. 1991) followed by thawing and recovery on MS media containing different hormone combinations (Harding and Benson 1994). Recovery of potato plants as time courses for in vitro regrowth and maturation were constructed as reported by Harding and Benson (1994) and Harding (1997). Apical shoot cultures of wild Solanum species: S. phureja, $S$. brachycarpum, $S$. acaule, S. guerreroense, $S$. iopetalum and $S$. tuberosum cv 'Pentland Squire' were cryopreserved using alginate encapsulationdehydration (Benson et al. 1996). Genotypes of Ribes ciliatum (sensitive) and $R$. nigrum cv 'Ben More' (tolerant) were selected based on their differential responses to cryopreservation (Reed et al. 2005). In vitro shoots were grown on MS-Ribes medium and shoot meristems sucrose-acclimated
(Johnston et al. 2007) and cryopreserved using encapsulation-dehydration (Reed et al. 2005). Stress marker profiles were constructed using antioxidants and volatile hydrocarbon analyses of methane (a marker for ${ }^{\bullet} \mathrm{OH}$ ) and ethylene (Johnston et al. 2007). Profiles were constructed during sucrose-simulated acclimation and after cryostorage.

\section{Results}

\section{Potato genotypic effects}

Differential genotype responses were observed in S. tuberosum cvs 'Golden Wonder' and 'Desiree', with 'Golden Wonder' being consistently more tolerant to cryopreservation irrespective of light or cryogenic treatments (Fig. 1). Both genotypes exhibited a progressive light-independent decline during recovery, with an onset at week 3 for cvs 'Golden Wonder', whereas 'Desiree' had consistently low levels of recovery across all light conditions (Fig. 1A). Light treatments had a significant effect on total recovery (mean $\%$ values) of both cvs 'Desiree' $(p=0.001, \mathrm{SEM}=1)$ and 'Golden Wonder' $(p=0.025, \mathrm{SEM}=2.9)$. A very significant interaction was observed (Fig. 1B) between freezing method, post-light treatment and time of total recovery (mean \% values) of cv 'Golden Wonder' ( $p=0.001$, $\mathrm{SEM}=4$ ). Overall, ultra-rapid freezing supported higher levels of recovery across both genotypes and high light triggered a decline in recovery and regrowth from week 3 onwards.

\section{Effects of in vitro age}

Age of culture (Fig. 2A) had a very significant effect on total recovery (mean \% values) in both cvs "Desiree" ( $p=0.001)$ and 'Golden Wonder' $(p=0.001)$. Higher survival was observed in younger (short-term) cultures cryopreserved within 6-8 weeks of their initiation in culture from tuber sprouts. Survival was reduced in older (long-term) 
Vol. 18 (2009): 103-116.

cultures maintained for 3 years. Young cultures of both cultivars performed better with respect to shoot production in surviving meristems (Fig. 2B). Culture age had a very significant effect $(p=0.001)$ on
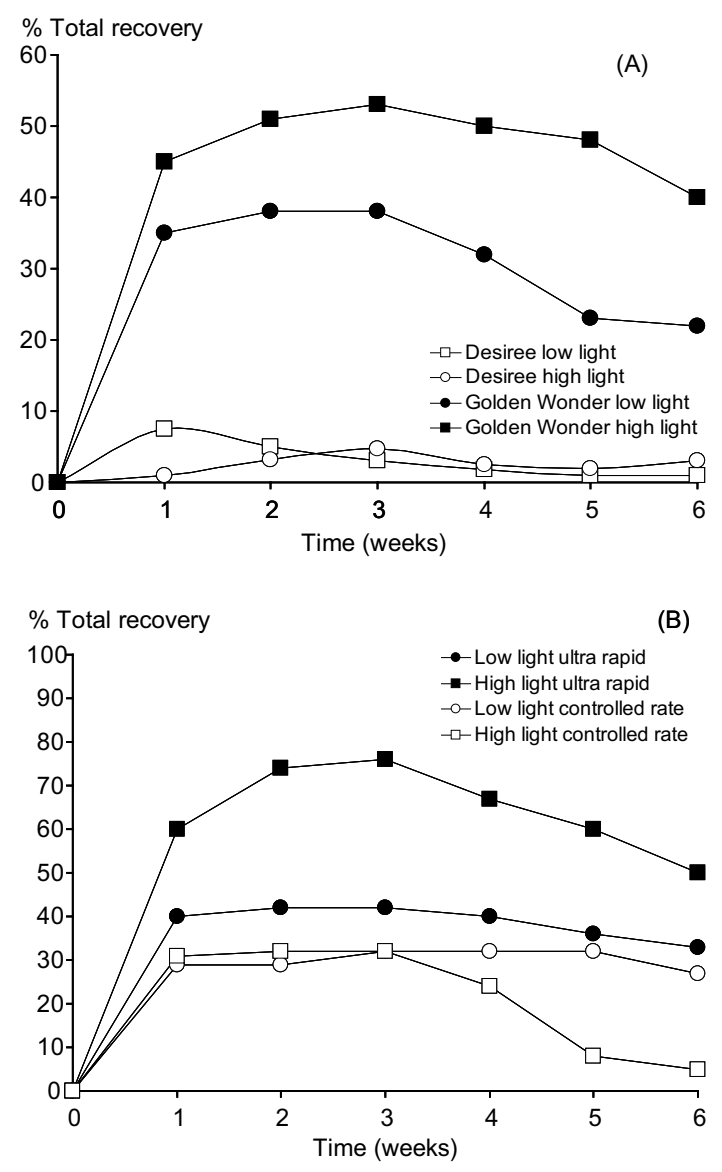

Fig. 1. Time course of (A) the effects of post-light on total recovery of cryopreserved shoot tips of $S$. tuberosum cvs 'Desiree' and 'Golden Wonder' and (B) the interaction between freezing method, post-light and time on total recovery of $S$. tuberosum cv 'Golden Wonder'. Data represented as mean (\%) values, probability $(p)$ and standard error of the mean (SEM) of three replicated experiments each of 25 shoot tips per light treatment. Low light treatment refers to photosynthetically active radiation (PAR) $15 \mu \mathrm{E} \cdot \mathrm{m}^{-2} \cdot \mathrm{sec}^{-1}$ and high light to PAR $45 \mu \mathrm{E} \cdot \mathrm{m}^{-2} \cdot \mathrm{sec}^{-1}$ original data from Benson et al. (1989) with permission of CryoLetters. cv 'Golden Wonder' for which meristem regrowth from younger cultures (Fig. 2B) occurred more rapidly and with a higher $\%$ regeneration compared to older cultures.

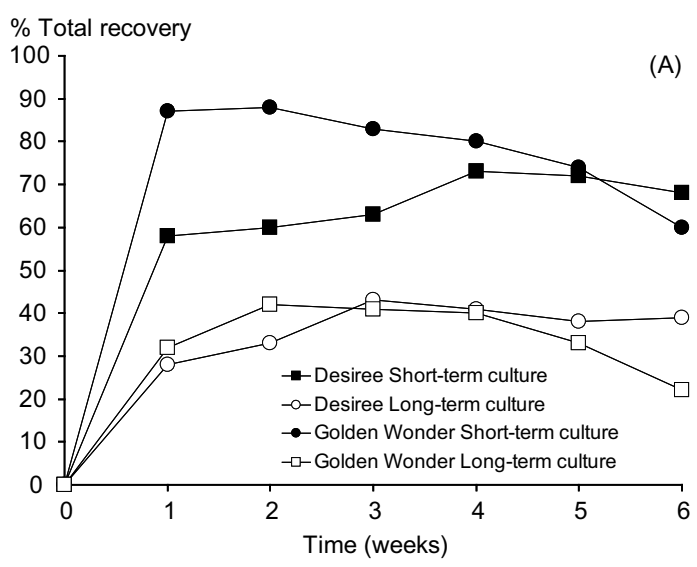

$\%$ Shoot regeneration

(B)

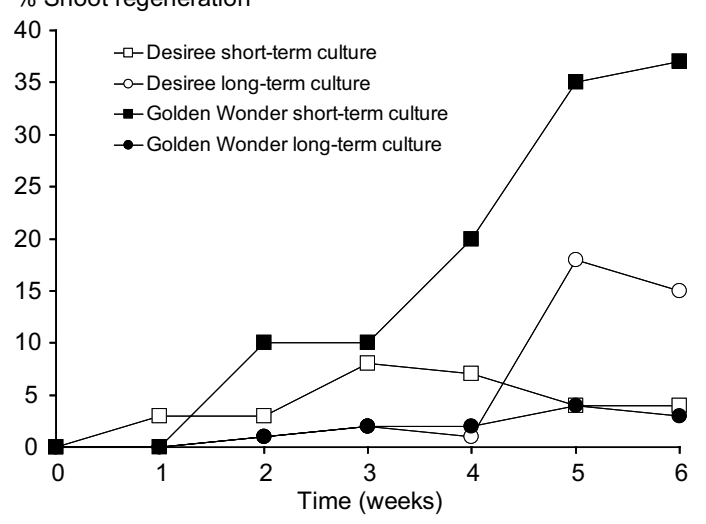

Fig. 2. The effect of pre-freeze short- (6-8 weeks old in vitro cultures) and long-term ( $\sim 3$ year old in vitro cultures) culture time on (A) total recovery of cryopreserved shoot tips of S. tuberosum cvs 'Desiree' and 'Golden Wonder' and (B) shoot regeneration of S. tuberosum cvs 'Desiree' and 'Golden Wonder'. Data represented as mean (\%) values and probability $(p)$ of 20 replicate plates derived from 100 shoot tips per treatment original data from Harding et al. (1991) with permission of CryoLetters. 
Harding, K. Exploring cryopreservation in clonally propagated crops

\section{Experimental variability}

Significant variation between triplicate experiments and replicate culture plates within an experiment were observed for cv 'Golden Wonder' shoot tips cryopreserved using ultra rapid freezing (Fig. 3). In sequence, variation (mean $\%$ values) was observed for total recovery ( $p=0.001$, SEM=3.2), shoot regeneration $(p=0.001, \mathrm{SEM}=1.4)$ following 6 weeks of culture and plantlet formation ( $p=0.05, \mathrm{SEM}=1.6$ ) over 12 months. Encapsulation-dehydration of shoot tips excised from in vitro cultures of wild Solanum species resulted in wide variation between triplicate experiments and replicates (mean \% values) with respect to total recovery and shoot regeneration after cryopreservation (Fig. 4).

\section{Dynamics of recovery and molecular analysis of potato}

Growth regulator composition affected growth and development during recovery of cv 'Golden Wonder' shoot tips after cryopreservation (Fig. 5A-C). TBZ medium proved to be the most successful supporting a more synchronized and uniform pattern of recovery. The sequential stages of recovery as mean times for: (i) in vitro growth (ii) plant maturity and (iii) total time to regenerate and mature with mean (\%) regeneration data for cv 'Golden Wonder' are shown in Table 1. Data ranked in order of increasing total recovery time show approximately a 100 day difference in recovery between media containing different hormones. Differences in DNA extracted from mature $S$. tuberosum plants are presented in Figure 5D as an autoradiograph of a Southern blot of the ribosomal RNA gene (rDNA) fragments detected using a $4.4 \mathrm{~kb}$ ribosomal probe derived from pTa 71. DNA samples (A-M) correlate respectively with plants recovered from cryopreserved shoot tips of cvs 'Golden Wonder' and 'Desiree' that had been recovered on different hormone regimes. The inset (arrow) marks the variable $2.55 \mathrm{~kb}$ rDNA fragment in a number of samples (B, D, F, I, J and L) and their respective densities are shown in Table 2 . A reduction in the $2.55 \mathrm{~kb}$ fragment density was

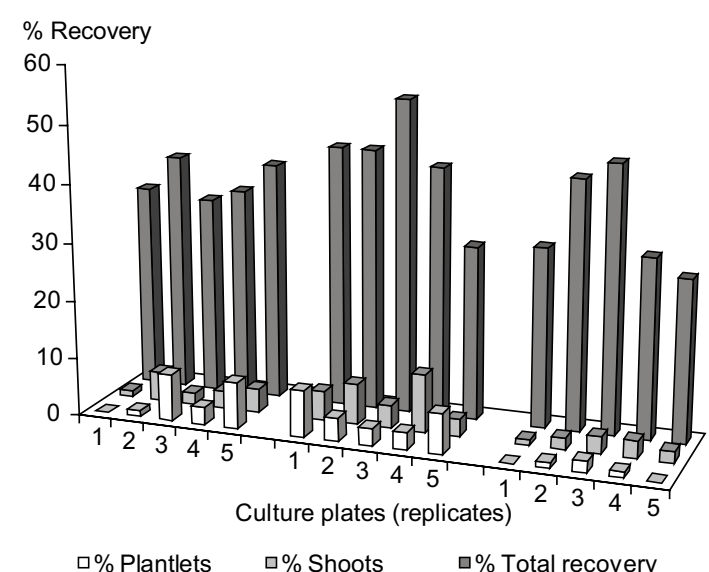

Fig. 3. Variation in total recovery, shoot regeneration and plantlet formation between triplicate experiments and replicate culture plates within an experiment of cryopreserved shoot tips of S. tuberosum cv 'Golden Wonder'. Data represented as mean (\%) values, probability $(p)$ and standard error of the mean (SEM) of three replicated experiments each of 25 shoot tips original data from Benson et al. (1989) with permission of CryoLetters.

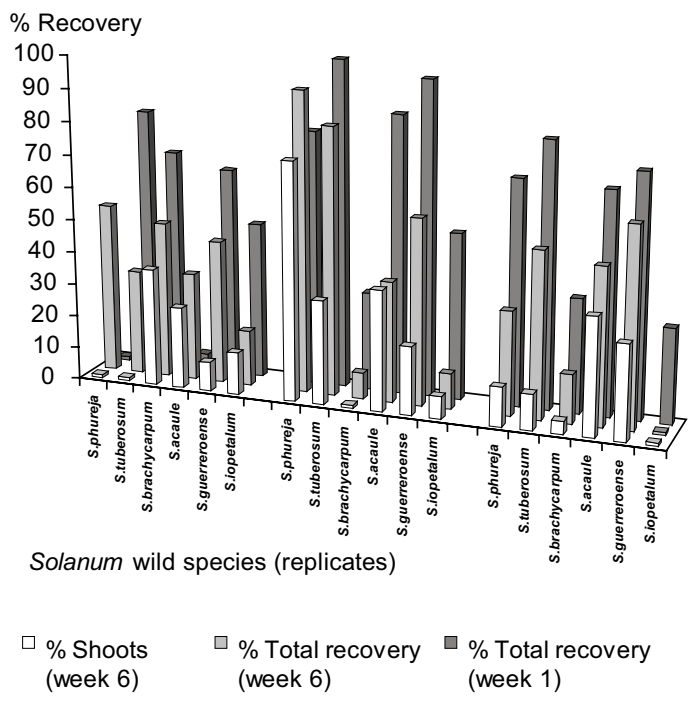

Fig. 4. Variation in total recovery and shoot regeneration between triplicate experiments and replicate samples within an experiment of cryopreserved shoot tips of diverse wild Solanum species. Data derived from three replicated experiments, where $\mathrm{n}=25-40$ shoot tips per replicate original data from Benson et al. (1996) with permission of CryoLetters. 
Vol. 18 (2009): 103-116.

observed in samples B, D, F, I, J and L (shown in bold) corresponding to $>2.00$ ratio for the other (3.1, 1.7 and $0.65 \mathrm{~kb})$ rDNA fragments. The respective positions for recovery of samples E, F, I and
$\mathrm{J}$ during in vitro culture (A), their maturation (B) and total time to regenerate and mature (C) are marked in Figure 5.

Table 1. In vitro culture time, maturation time of plantlets, total time of plants to (\%) regenerate and mature following cryopreservation of S. tuberosum cvs Golden Wonder and Desiree shoot tips recovered on media containing different hormone regimes.

\begin{tabular}{lcccc}
\hline Media & In vitro time & Maturation time & Total time & Regeneration (\%) \\
\hline Golden Wonder & 94 & 108 & 202 & 42.7 \\
TBZ & 132 & 108 & 240 & 12.0 \\
GA3 & 142 & 109 & 252 & 13.3 \\
HF-BSA & 159 & 109 & 269 & 12.0 \\
NAA/GA3 & 132 & 170 & 302 & 2.7 \\
GA3-BSA & 177 & 126 & 303 & 17.3 \\
HF & 189 & 125 & 314 & 2.7 \\
GA3/IAA & 118 & 104 & & 2.0 \\
Desiree & 126 & 97 & 222 & 0.7 \\
GA3/IAA & 132 & 98 & 231 & 2.7 \\
HF-BSA & 164 & 120 & 284 & 2.7 \\
GA3 & 169 & 117 & 287 & 3.3 \\
TBZ & 202 & 101 & 304 & 1.3 \\
NAA/GA3 & 164 & 318 & 1.3 \\
HF & & & & \\
GA3-BSA & & & & \\
\hline
\end{tabular}

Table 2. Ribosomal RNA gene fragment densities/ratios for cryopreserved S. tuberosum samples

\begin{tabular}{lccccccccccccc}
\hline $\begin{array}{l}\text { Fragment } \\
\text { size }(\mathrm{kb})\end{array}$ & \multicolumn{10}{c}{ Samples (density of fragments) } \\
\cline { 2 - 14 } & $\mathrm{A}$ & $\mathrm{B}$ & $\mathrm{C}$ & $\mathrm{D}$ & $\mathrm{E}$ & $\mathrm{F}$ & $\mathrm{G}$ & $\mathrm{H}$ & $\mathrm{I}$ & $\mathrm{J}$ & $\mathrm{K}$ & $\mathrm{L}$ & $\mathrm{M}$ \\
\hline 3.1 & 0.70 & 0.78 & 0.88 & 0.66 & 0.94 & 0.84 & 0.93 & 1.07 & 0.87 & 0.86 & 0.95 & 0.84 & 0.91 \\
$\mathbf{2 . 5 5}$ & $\mathbf{0 . 5 2}$ & $\mathbf{0 . 3 5}$ & $\mathbf{0 . 8 4}$ & $\mathbf{0 . 3 3}$ & $\mathbf{0 . 6 9}$ & $\mathbf{0 . 1 7}$ & $\mathbf{0 . 7 7}$ & $\mathbf{0 . 8 2}$ & $\mathbf{0 . 2 6}$ & $\mathbf{0 . 3 0}$ & $\mathbf{0 . 7 2}$ & $\mathbf{0 . 1 3}$ & $\mathbf{0 . 7 0}$ \\
1.70 & 0.65 & 0.70 & 0.82 & 0.57 & 0.72 & 0.54 & 0.66 & 0.68 & 0.66 & 0.62 & 0.67 & 0.68 & 0.75 \\
0.65 & 0.86 & 0.91 & 1.02 & 0.89 & 0.94 & 0.90 & 0.94 & 1.14 & 0.96 & 0.90 & 0.98 & 0.92 & 0.98 \\
& & & & & & Samples (ratios) & & & & & \\
3.1 & 1.35 & $\mathbf{2 . 2 3}$ & 1.05 & $\mathbf{2 . 0 0}$ & 1.36 & $\mathbf{4 . 9 0}$ & 1.21 & 1.31 & $\mathbf{3 . 3 0}$ & $\mathbf{2 . 9 0}$ & 1.31 & $\mathbf{6 . 4 6}$ & 1.30 \\
2.55 & & & & & & & & & & & & & \\
1.70 & 1.25 & $\mathbf{2 . 0 0}$ & 0.98 & $\mathbf{1 . 7 2}$ & 1.04 & $\mathbf{3 . 2 0}$ & 0.86 & 0.83 & $\mathbf{2 . 5 1}$ & $\mathbf{2 . 1 0}$ & 0.93 & $\mathbf{5 . 2 3}$ & 1.07 \\
0.65 & 1.65 & $\mathbf{2 . 6 0}$ & 1.21 & $\mathbf{2 . 7 0}$ & 1.36 & $\mathbf{5 . 3 0}$ & 1.22 & 1.39 & $\mathbf{3 . 9 6}$ & $\mathbf{3 . 0 0}$ & 1.36 & $\mathbf{7 . 0 8}$ & 1.40 \\
\hline
\end{tabular}

Original data from Harding (1997) re-used with CryoLetter's permission. 
Harding, K. Exploring cryopreservation in clonally propagated crops
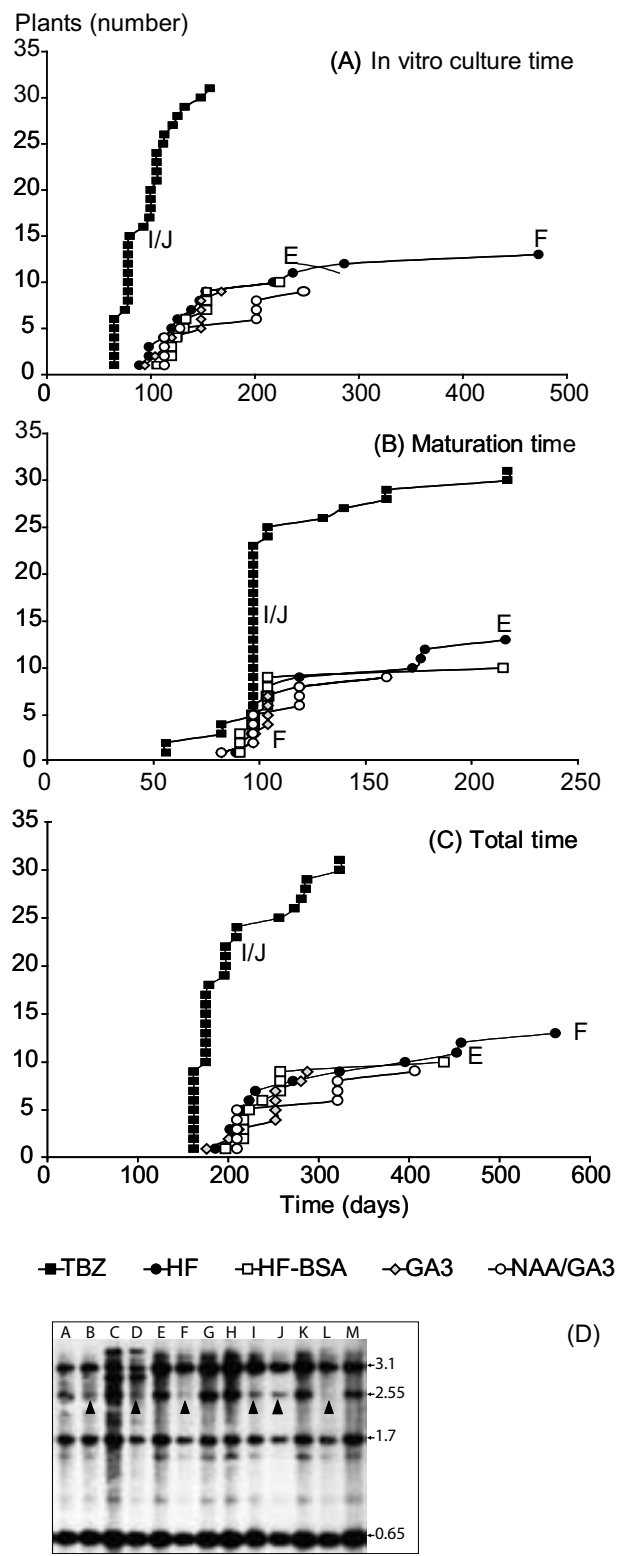

(D)

Fig. 5. In vitro culture time of plantlets (A), maturation time of plantlets (B) and total time of plants to regenerate and mature $(\mathrm{C})$ and ribosomal RNA gene fragments detected in $S$. tuberosum plants (A-M) recovered on media containing different hormone regimes (D) following cryopreservation. Data represented as mean (\%) values of three replicated experiments each of 25 shoot tips per treatment for recovery media: HF, HF-BSA, GA3, GA3/NAA and TBZ original data from Harding and Benson (1994) and Harding (1997) with permission of CryoLetters.

\section{Recovery and oxidative stress in Ribes genotypes}

The recovery of sucrose-acclimated Ribes shoot tips demonstrates $R$. nigrum is more tolerant to cryopreservation, whereas the time course shows a progressive decline in total recovery for the $R$. ciliatum genotype (Fig. 6). Following exposure to successive stages of the encapsulation-dehydration protocol indicates variable stress responses with differential shoot regrowth between the sensitive (R. ciliatum) and tolerant ( $R$. nigrum) genotypes (Fig. 7 inset). Markers for oxidative stress and antioxidant status (Table 3) revealed significant differences between the two genotypes which can be delineated into two stress profiles (Fig. 7).

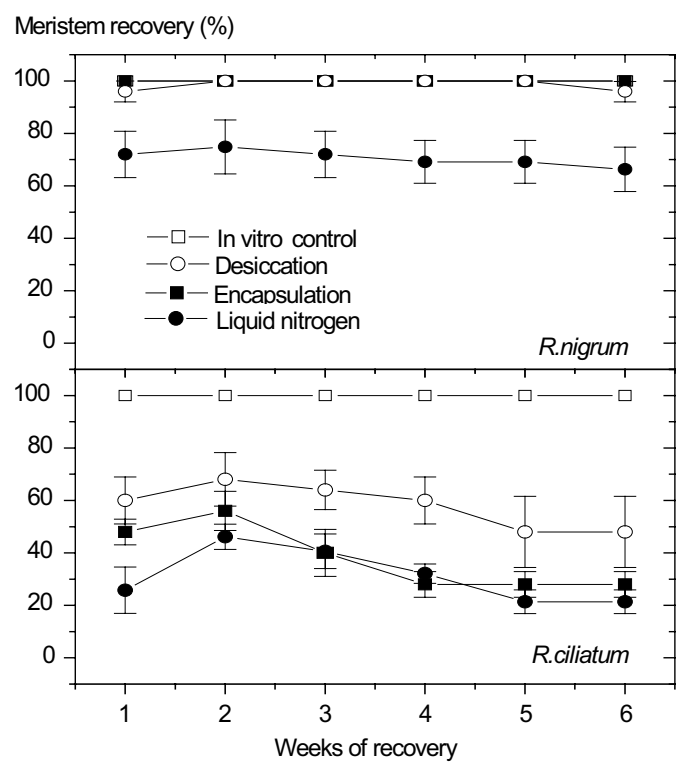

Fig. 6. Time course of cryopreservation tolerant (R. nigrum cv Ben More) and sensitive ( $R$. ciliatum) shoot tips recovering from different stages of encapsulation-dehydration following 1-2 weeks (as unbleached shoots), 3-6 weeks (as green meristems and expanded leaves) of culture. Values (\%) are means and standard errors of the means, where $n=5$ replicates with 5-8 meristems per replicate original data from Johnston et al. (2007) with permission of Elsevier. 
Vol. 18 (2009): 103-116.

Table 3. Oxidative stress markers and antioxidant status in Ribes genotypes following acclimation and cryopreservation.

\begin{tabular}{|c|c|c|c|c|}
\hline \multirow{2}{*}{ Stress markers \& antioxidants } & \multicolumn{2}{|c|}{ R. ciliatum (sensitive) } & \multicolumn{2}{|c|}{ R. nigrum (tolerant) } \\
\hline & Acclimation & $\mathrm{LN}$ & Acclimation & $\mathrm{LN}$ \\
\hline Chlorophyll $\mathrm{a}^{1}$ & $47.6 \pm 2.3$ & - & $67.1 \pm 5.6$ & - \\
\hline Chlorophyll $\mathrm{b}^{1}$ & $25.5 \pm 1.0$ & - & $45.4 \pm 3.7$ & - \\
\hline Chlorophyll a:b ratio & $1.9 \pm 0.1$ & - & $1.5 \pm 0.1$ & - \\
\hline Carotenoids $^{1}$ & $21.2 \pm 2.1$ & - & $31.1 \pm 2.4$ & - \\
\hline Methane $^{3}$ & $11.4 \pm 1.5$ & $11.0 \pm 5.8$ & $33.6 \pm 9.0$ & $12.9 \pm 5.1$ \\
\hline Ethylene $^{3}$ & $4.5 \pm 0.7$ & $42.5 \pm 6.8$ & $6.2 \pm 3.1$ & $14.6 \pm 4.7$ \\
\hline Total protein $+\mathrm{PVPP}^{1}$ & $3.83 \pm 0.4$ & $4.8 \pm 0.2$ & $1.1 \pm 0.2$ & $0.5 \pm 0.4$ \\
\hline Total protein - $\mathrm{PVPP}^{1}$ & $1.9 \pm 0.2$ & $2.6 \pm 0.7$ & $0.7 \pm 0.1$ & $1.8 \pm 0.5$ \\
\hline Non-phenolic antioxidants ${ }^{2}$ & $5.6 \pm 0.3$ & $2.4 \pm 0.1$ & $11.7 \pm 0.3$ & $4.6 \pm 0.7$ \\
\hline Total antioxidants ${ }^{2}$ & $29.8 \pm 2.2$ & $6.5 \pm 1.1$ & $101.6 \pm 7.3$ & $65.9 \pm 10.9$ \\
\hline Total phenolics ${ }^{1}$ & $19.6 \pm 0.4$ & $5.8 \pm 0.5$ & $52.4 \pm 2.3$ & $53.3 \pm 8.1$ \\
\hline Protein $\mathrm{SH}^{2}$ & $0.2 \pm 0.1$ & $1.7 \pm 0.1$ & $0.4 \pm 0.2$ & $2.5 \pm 0.1$ \\
\hline Non-Protein $\mathrm{SH}^{2}$ & $0.8 \pm 0.1$ & $0.2 \pm 0.1$ & $1.6 \pm 0.1$ & $0.4 \pm 0.1$ \\
\hline Total $\mathrm{SH}^{2}$ & $0.9 \pm 0.1$ & $2.2 \pm 0.3$ & $1.9 \pm 0.2$ & $2.9 \pm 0.1$ \\
\hline
\end{tabular}

Summary data of oxidative and photooxidative (as pigments) stress markers, proteins and antioxidant levels (units: 1 - mg.g FW FW $^{-1} 2-$ $\mu$ mol.g FW ${ }^{-1} ; 3$ - pmol.s.kg $\left.{ }^{-1}\right)$ of Ribes sensitive and tolerant genotypes following sucrose-simulated cold acclimation $(7$ days, $0.75 \mathrm{M}$ Suc-RIB medium) and cryopreservation (LN) using cryoprotective encapsulation-dehydration ( $\mathrm{PVPP}=$ polyvinylpolypyrrolidone, $\mathrm{SH}$ $=$ sulphydryl groups). Original data from Johnston et al. (2007) kindly re-used with Elsevier's permission.

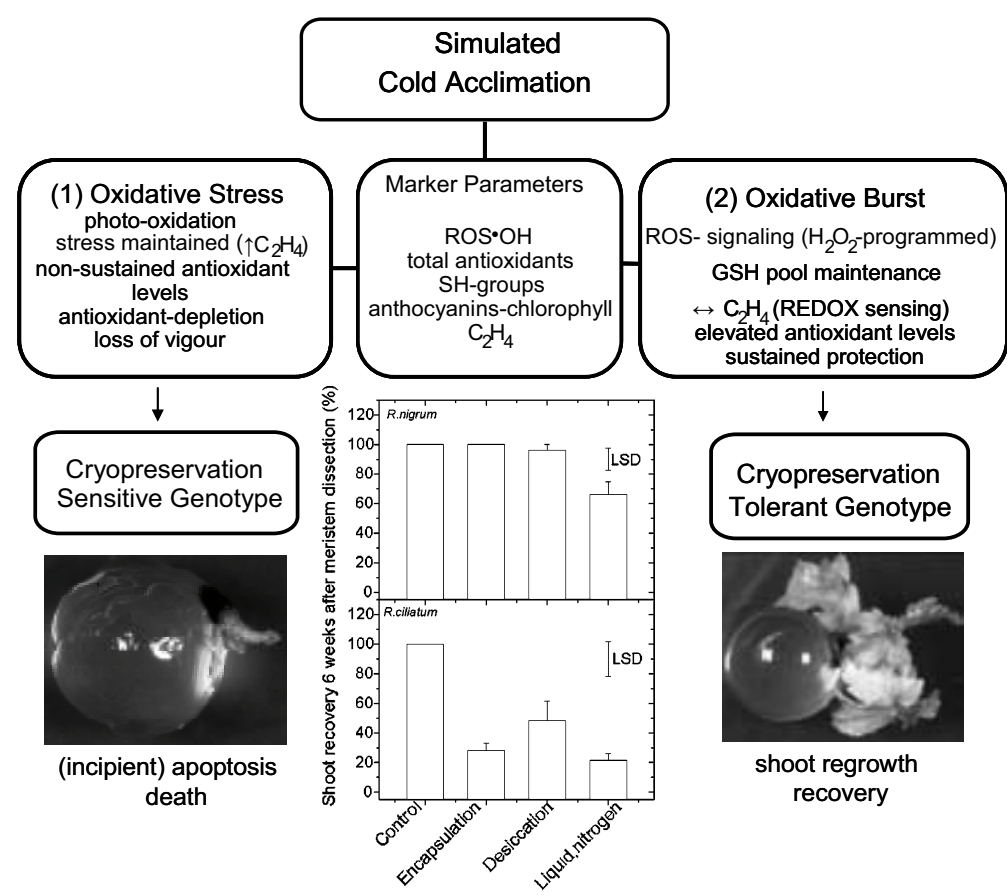

Fig. 7. Model of Ribes responses to cryopreservation based on oxidative stress and antioxidant markers. Insert graphic: shoot production of $R$. nigrum (cv Ben More) and R. ciliatum meristems recovered on RIB medium, as a percentage (\%) of meristems regenerating morphologically normal shoots 6 weeks following exposure to different stages of cryopreservation. Values are means $(\mathrm{n}=$ 5 replicates, 5-8 meristems per replicate), standard errors of the mean and $5 \%$ (LSD) least significant differences. $P$ values for stages of the protocol $R$. nigrum $(p=<0.001)$ and $R$. ciliatum $(p=<0.001)$ original data from Johnston et al. (2007) with permission of Elsevier. 
Harding, K. Exploring cryopreservation in clonally propagated crops

\section{Discussion}

Significant progress in protocol development has resulted in cryopreservation being more widely adopted in genebanks (Kim et al. 2006, Reed 2008) however, for some germplasm variable responses and recalcitrance remain limiting factors affecting cryobank efficiency. Some variability is attributed to known operational factors (Reed et al. 2001, 2004, 2005) and minor, often unpublished technical details (Keller et al. 2008). This comparative study of Solanum and Ribes also suggests that genotype, protocol, culture regime (light and growth regulator), age of culture and intrinsic experimental variation influence meristem survival and development following cryopreservation. This concurs with previous reports (Schäfer-Menuhr et al. 1997, Golmirzaie and Panta 2000, Keller 2005, Reed et al. 2005).

\section{Predetermined and genotype factors}

The droplet (PVS2) vitrification protocol (Panis et al. 2005, Kim et al. 2006) is proving widely applicable across a diverse range of crops, genotypes and cultivars (Sakai and Engelmann 2007). However, variable (intra/inter-species) genotypic responses to cryopreservation can limit its use to certain crops for which widely applicable, validated protocols are desirable. Successful protocols have been largely developed by empirical means however, proteomics now reveals tolerance to cryopreservation has a molecular-physiological basis (Carpentier et al. 2007). Where, genotype is a determinant in recalcitrant germplasm the use of suboptimal protocols may exacerbate and contribute to cryopreservation failure. Comparisons of recovery in $S$. tuberosum cvs 'Desiree' and 'Golden Wonder' (Figs. 1, 2) consistently show 'Desiree' is less amenable to cryopreservation across treatments. Similarly, other studies of cv 'Desiree' demonstrate different survival/shoot (\%) responses including: controlled rate freezing $\sim 2 / 0 \%$ (Benson et al. 1989), ultra-rapid freezing $\sim 55 / 10 \%$ (Harding et al. 1991), droplet-freezing $\sim 41 / 25 \%$ (Schäfer-Menuhr et al. 1997), 33/21.7\%
(Kryszczuk et al. 2006) and 10-21.4/0\% (Keller and Dreiling 2003), encapsulation-dehydration $78.8 / 58.8 \%$ (Grospietsch et al. 1999) and vitrification 70/38.4\% (Kryszczuk et al. 2006). Although, protocol refinement enhanced survival this did not necessarily convert to increased development of shoots and plantlets in cv 'Desiree'. This effect was also observed in wild species of potato following shoot cryopreservation using encapsulation-dehydration (Fig. 4) suggesting it may be due to intrinsic stress factors. This is supported by Martinez et al. (2001) who conducted a comparative study on frost sensitive $S$. tuberosum and frost resistant $S$. curtilobum showing higher antioxidant activity (as superoxide dismutase) in the resistant native Andean potato species to freezing and drought. Studies of frost tolerant and intolerant Norway spruce (Picea abies) also revealed pre-existing high levels of antioxidants in seedlings contributed to enhanced tolerance in the frost-resistant genotype (Blödner et al. 2005). Cultivars of black currants are distinguished for breeding purposes by their differential sensitivities to spring frosts (Keep et al. 1983) and this helps the selection for intrinsic frost resistance genetic traits in the crop (Mather et al. 1980). The cryopreservation tolerant $R$. nigrum cv 'Ben More' used in this study (Figs. 6, 7) has been previously tested as flower bud frost resistant by Dale and Heiberg (1984). Comparisons of oxidative stress and antioxidants of $R$. ciliatum and $R$. nigrum ('Ben More') similarly suggest differential responses are genetically predetermined. Furthermore, wild and cultivated Ribes genotypes display variable recovery responses ranging from zero survival to $90 \%$ shoot regrowth following exposure of meristems to liquid nitrogen (Reed et al. 2001, 2004, 2005).

\section{In Vitro Physiology}

It is vitally important to recognise that cryopreservation success falls into two distinct phases: (1) the initial shoot tip survival, i.e. viability and (2) the ability of surviving meristems to develop shoots and plantlets. Lack of regrowth often contributes to cryostorage failure and this has implications for 
Vol. 18 (2009): 103-116.

genebank efficiency. The arrested growth of meristems is associated with inherent adaptations induced by cryogenic and in vitro treatments. For potato, cryopreservation may stimulate genotype dependent dormancy which impairs meristem development during recovery. This is observed in various growth regulator and additive (BSA) treatments applied to cvs 'Golden Wonder' and 'Desiree'. Developmental time courses (Fig. 5, Table 1) were strongly influenced by recovery medium with differences of ca. 100 days in the mean time taken for plantlets to regenerate and mature (Harding and Benson 1994, Harding 1997). The dynamics of recovery and significance of physiological uniformity is manifest as synchronicity between individual potato plants regenerated within the same batch of cryopreserved meristems (Fig. 5). Recovery on TBZ medium produced the most survivors, developed the most shoots and matured the most plants, within the shortest time. In contrast, recovery on hormone free (HF) medium supported a low number of survivors, shoots and plantlets demonstrating post-cryopreservation development can be greatly improved by growth regulators. Pre- and post- cryopreservation light treatments were also found to influence survival and shoot development in recovering cryopreserved potato meristems (Fig. 1). Thus, non-cryogenic factors can be optimized to enhance survival and development after cryopreservation. Other hitherto unknown factors may also contribute to differences between shoot meristems and plant culture cycles accounting in part for inter-experimental and intrareplicate variability (Fig. 3). Similar observations are reported for the initial survival, regrowth and plantlet development of garlic following cryopreservation (Keller 2005). Differences in physiological status of meristems may also explain variation between experiments and replicate samples (Fig. 4 ) in diverse potato species (Golmirzaie and Panta 2000) and old varieties of potato (Schäfer-Menuhr et al. 1997). The basis of these outcomes in clonally propagated crops such as potato remains speculative but cumulative evidence suggests it is partly due to meristem physiology. Sensitivity of shoot tips from 1-2 month old in vitro potato plants to droplet-vitrification was related to physiological state. A progressive decline in recovery occurred with decreasing bud ranking order from the apex (Halmagyi et al. 2005). The significance of shoot location and subculture duration of the mother plant has been similarly noted by Yoon et al. (2006) who also used droplet vitrification. Whereas, little or no differences were observed with respect to nodal segments or days of culture for potato shoots cryopreserved using encapsulation/vitrification (Hirai and Sakai 1999). These observations illustrate that intrinsic variability in vitro cultures does not ensure a sufficient level of physiological uniformity in some donor germplasm.

\section{In Vitro Ageing}

Sultan (2000) proposes a single genotype can produce different phenotypes in response to different in vivo environments and Munné-Bosch (2007) suggests intrinsic changes during the ageing of perennials become fixed in development and this affects the physiology of the whole plant. These observations are relevant to in vitro tissue cultures maintained for long periods, as in vitro ageing may differentially affect their response to cryopreservation. Age strongly influences the survival and regeneration of cryopreserved potato meristems; this is independent of genotype as older cultures are less able to recover from cryostorage (Fig. 2). Long-term in vitro cultures of cv 'Desiree' introduced in 1980 were unable to regrow following cryopreservation (Keller and Dreiling 2003) and 'old' and 'young' in vitro garlic cultures respond differentially (Keller 2005). Prolonged culture in vitro is known to cause loss of totipotency, vigour and promote neoplastic progression (Benson 2000, Gaspar et al. 2002) illustrating that changes in explant quality as a result of in vitro ageing may exacerbate genotypic responses to cryopreservation. It is therefore, recommended younger, newly initiated cultures are preferentially selected for cryopreservation or older cultures are regenerated/rejuvenated before their meristems are cryobanked. 
Harding, K. Exploring cryopreservation in clonally propagated crops

\section{An Oxidative Stress Model for Different Survival Responses}

Recovery data of potato and Ribes (Figs. 1, 2, 6, 7) initially show high levels of survival in the more tolerant genotypes followed by a progressive decline which is more pronounced in sensitive genotypes. The recurrent loss of original survivors coupled with failure of those that are remaining to develop shoots results in substantial diminishing returns from the cryobank. Recovery and oxidative stress profiles of cryopreservation tolerant $R$. nigrum and sensitive $R$. ciliatum are markedly different and comprise two distinct phases (Johnston et al. 2007). Initially (1-2 weeks) up to $70 \%$ of $R$. ciliatum shoot meristems survived but by $3-5$ weeks most had perished and only $20 \%$ of those remaining developed shoots (Fig. $6)$. In contrast $R$. nigrum meristems maintained relatively high levels of recovery throughout cryopreservation, up to $70 \%$ of the meristems survived liquid nitrogen of which ca. $100 \%$ produced shoots (Fig. 6). Oxidative stress profiles (Table 3) also revealed different genotypic responses to acclimation and cryopreservation leading to the postulation that two different oxidative stress pathways occur in Ribes shoots recovering from cryopreservation (Fig. 7). The first involves a deleterious reaction to oxidative stress manifest as free radical mediated (photo) oxidation, failure of antioxidant protection, degeneration, death of early survivors and loss of morphogenetic competence. Alternatively, the second pathway comprises a beneficial, programmed oxidative burst, punctuated by enhanced ${ }^{\bullet} \mathrm{OH}$ production and the putative involvement of Reactive Oxygen Species (ROS) in anti-stress signaling. This results in an elevated antioxidant status, enhanced tolerance to cryopreservation and the conversion of ca. $65-80 \%$ of the cryopreserved meristems to shoots (Fig. 7). These differential responses were initiated during sucrose-simulated cold acclimation and subsequent tolerance to liquid nitrogen correlated to increases in ${ }^{\bullet} \mathrm{OH}$ activity, $\mathrm{C}_{2} \mathrm{H}_{4}$ evolution, antioxidant status, phenolic accumulation, protein $\mathrm{SH}$ group status and a reduction in chlorophyll and carotenoid photooxidation as compared to the sensitive genotype (Table 3, Fig. 7). Importantly, antioxidant protection in $R$. nigrum persisted during recovery indicating elevated antioxidant status is associated with cryopreservation tolerance (Johnston et al. 2007). These findings concur with in vivo studies of freezing stress in which antioxidant protection acquired during cold acclimation and drought tolerance is associated with genotypic tolerance to freezing (Blödner et al. 2005, Dale and Heiberg 1984, Mather et al. 1980).

\section{Is Programmed Cell Death a Factor in Delayed Post-Cryopreservation Failure?}

Baust (2002) proposes delayed onset, cryopreservation-induced cell death as a significant contributory factor in storage failure and recommends that apoptosis and necrosis should be considered when developing biopreservation protocols. As a delayed decline in recovery has been observed in plant meristems following cryopreservation (Figs. 1, 6) there may be some parallels with animal tissue cryostorage. Cell death incurred by cryoinjury is known to be associated with a belated free radical cascade in human tissues (Hoffman and Bischoff 2004) and apoptosis is induced via the release of cytochrome $c$ from mitochondria (Reape and McCabe 2008). Thus, oxidative stress and apoptosis may become causal and contributory factors in predisposing both plant and animal tissues to immediate and delayed onset cryoinjury. In evaluating programmed cell death in plant cryopreservation it is important to differentiate between events that lead to necrosis and those that are confirmed as truly apoptotic by using biochemical markers (Bischof et al. 2002, Gage and Baust 2002, Reape et al. 2008). As apoptosis remains to be fully elucidated in plants, it is also necessary to distinguish between necrosis and apoptosis-like programmed cell death during cryostorage recovery responses (Reape and McCabe 2008). Nevertheless, understanding the mechanisms involved is important as degenerative responses are often observed in storage sensitive genotypes and the delayed loss of survivors can be sufficiently significant to affect the efficiency of plant genebank operations. The fact that the delayed 
Vol. 18 (2009): 103-116.

decline in meristem survival is frequently independent of protocol suggests cryopreservation stresses per se may exacerbate degenerative pathways, some of which proceed via necrosis, whilst others may involve programmed cell death (Reape et al. 2008). In the case of certain genotypes, optimisation of cryogenic factors may not be sufficient and other strategies will be needed to ensure sustained recovery. These are likely to include the manipulation of physiological status prior to cryopreservation, acclimation treatments, choosing vigorous, young donor cultures and excising meristems in an appropriate stage of development. Exploring apoptotic induction factors in both immediate and delayed onset post-storage degeneration in plant germplasm has practical merits particularly as it is under investigation in other biorepository sectors (Khan et al. 2009, Baust 2002).

\section{Cryoinjury, Genetic Stability and Cryobio- nomics}

Physiological factors are likely to contribute to variable responses independent of the cryopreservation protocol and an investigative molecular approach may help elucidate the basis for these differences. Figure 5D and Table 2 show rDNA fragment variability within a given batch of individual cryopreserved plants providing further support for the premise that physiological variation occurs during in vitro manipulation (Harding et al. 2008, Carpentier et al. 2007). Ribosomal fragment variability possibly results from restriction enzyme sensitivity to methylation of enzyme recognition sites (Harding 1997). DNA methylation as an epigenetic mechanism for genomic variability (Harding 2004, Harding et al. 2008) may also account for physiological variation between individual meristems and their differential responses (Johnston et al. 2007, Johnston et al. 2009). Although, in vitro variation within batches of individual meristems clearly exists, it is not inherently obvious prior to cryopreservation and this makes its detection difficult. The use of DNA methylation as an epigenetic marker for physiological variability may be useful in evaluat- ing recalcitrant germplasm before cryopreservation (Johnston et al. 2005, Harding et al. 2008, Johnston et al. 2009). There is evidence that differences between plants showing differential methylation affect their regeneration and maturation following cryopreservation (Fig. 5, Harding et al. 2008). The time taken for in vitro plantlets $(\mathrm{E}, \mathrm{F})$ to regenerate is also related to differential rDNA methylation as evidenced by the delays incurred on hormone free recovery medium compared to plantlets (I, J) with similar levels of methylation and recovery times (Fig. 5A) using more complex medium (Harding 1997). Although, the respective positions for the plantlets (I, J) are relatively unchanged (Fig. 5A, B) others (E, F) show the opposite trend in recovery times between growth in vitro and maturation (Harding and Benson 1994). Clearly, there are complex interactions between the epigenetic regulation of gene expression and hormone induced physiological responses in these plants.

Collective evidence illustrates the intricate combined effects of cryogenic and non-cryogenic factors following the exposure of shoot meristems to the physical, chemical and physiological stresses that cause cryoinjury (Johnston et al. 2007, Johnston et al. 2009). The effects of cryoinjury upon the genome are often unknown; any accumulative DNA polymorphisms may not be induced by solely cryogenic factors but result from the whole process (Harding 2004). The intrinsic cellular and metabolic factors (Carpentier et al. 2007, Harding et al. 2008) that affect physiological status can profoundly determine meristem survival. For example, where, survival is mostly dependent on antioxidant defences that oppose the deleterious effects of cryoinjury (Johnston et al. 2007). The hypothetical relationship between cryoinjury, genetic stability and cryobionomics is shown in Figure 8 which highlights the potential impacts of cryoinjury on the genome, transcriptome, proteome and metabolome. This leads to several possible changes, those incurred by modulated patterns of gene expression mediated through epigenetic mechanisms because of naturally adaptive responses, as well as events that lead to undesirable instability. These may be manifest as detectable changes that affect truenessto-type with respect to morphology, histology, cy- 
Harding, K. Exploring cryopreservation in clonally propagated crops

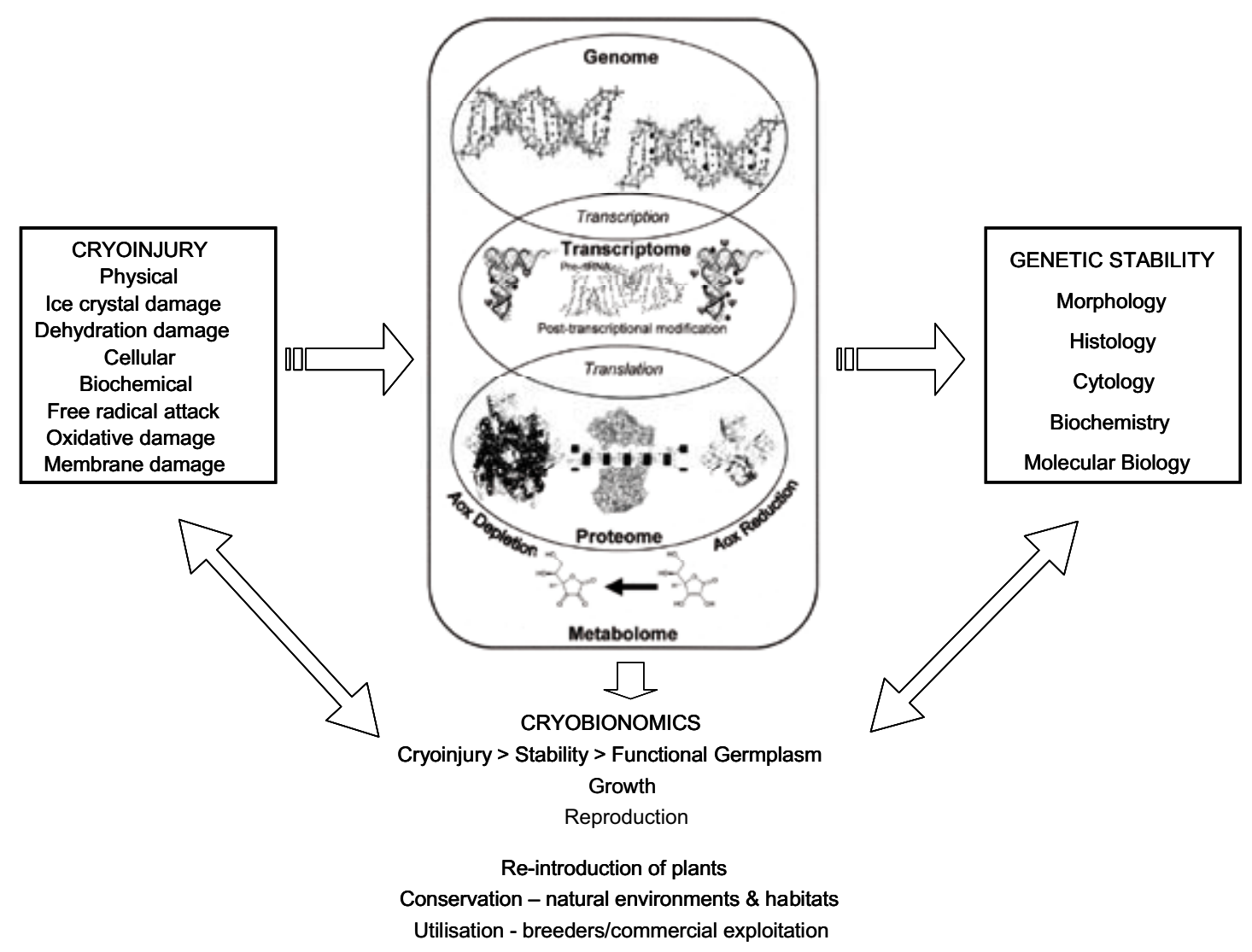

Fig. 8. Relationship between cryoinjury, genetic stability and cryobionomics.

tology, biochemistry and the molecular biology of whole plants recovered from cryopreserved germplasm (Harding 2004). Profound epi- and/or genetic alterations to the genome considered within the framework of 'cryobionomics' are likely to affect the function of germplasm possibly by disrupting established patterns of growth and reproduction. These changes may influence both the quality of conserved germplasm and the re-introduction of plants recovered from cryobanked materials into natural environments and habitats, and including their utilisation by breeders and exploitation in commercial activities. This study recommends a more holistic approach is now required to understand the basis of success or failure following cryostorage. Cryobionomics provides a conceptual framework to explore the linkages between cryogenic and non-cryogenic stress factors by using molecularphysiological approaches to aid cryopreservation protocol improvement and optimization.

Acknowledgements: The Commission of the EU for CRYMCEPT project (QLK5-CT-2002-01279) and EU COST Action 871 CRYOPLANET, the USDA NCGR, Oregon, and SCRI for providing Ribes and potato germplasm. 
Vol. 18 (2009): 103-116.

\section{References}

Baust, J.M. 2002. Molecular mechanisms of cellular demise associated with cryopreservation failure. Cell Preservation Technology 1: 17-31.

Benson, E.E. 2000. Special symposium: In vitro plant recalcitrance do free radicals have a role in plant tissue culture recalcitrance? In Vitro Cellular \& Development Biology-Plant 36: 163-170.

Benson, E.E., Harding, K. \& Smith, H. 1989. Variation in recovery of cryopreserved shoot tips of Solanum tuberosum exposed to different pre- and post-freeze light regimes. CryoLetters 10: 323-344.

Benson, E.E., Wilkinson, M., Todd, A., Ekuere, U. \& Lyon, J. 1996. Developmental competence and ploidy stability in plants regenerated from cryopreserved potato shoot tips. CryoLetters 17: 119-128.

Bischof, J.C., Coad, J.E., Hoffman, N.E. \& Roberts, K.R. 2002. Is apoptosis an important mechanism of cryoinjury in vivo? CryoLetters 23: 277-278.

Blödner, C., Skroppa, T., Johnsen, Ø. \& Polle, A. 2005. Freezing tolerance in two Norway spruce (Picea abies $\mathrm{L}$. Karst.) progenies is physiologically correlated with drought resistance. Journal of Plant Physiology 162: 549-558.

Carpentier, S.C., Witters, E., Laukens, K., Van Onckelen, H., Swennen, R. \& Panis, B. 2007. Banana Musa spp. as a model to study the meristem proteome: acclimation to osmotic stress. Proteomics 7: 92-105.

Dale, A. \& Heiberg, N. 1984. Studies in black currant (Ribes nigrum L.) on the relationship between frost tolerance in winter and spring and on the relationships of dehardening and rehardening in spring. Crop Research 24: 73-83.

Gage, A. \& Baust, J.G. 2002. Reply to Dr John Bischof and colleagues from Dr Andrew Gage and Dr John Baust. CryoLetters 23: 279-280.

Gaspar, T., Franck, T., Bisbis, B., Kevers, C., Jouve, L., Hausman, J.F. \& Dommes, J. 2002. Concepts in plant stress physiology: Applications to plant tissue cultures. Plant Growth Regulation 37: 263-285.

Golmirzaie, A.M. \& Panta, A. 2000. Advances in potato cryopreservation at CIP. In: Engelmann, F. \& Takagi, H. (eds). Cryopreservation of Tropical Plant Germplasm. Current Research Progress and Application IPGRI, Rome. p. 250-254.

Grospietsch, M., Stodulková, E. \& Zámecnik, J. 1999. Effect of osmotic stress on the dehydration tolerance of Solanum tuberosum shoot tips. CryoLetters 20: 339-346.

Halmagyi, A., Deliu, C. \& Coste, A. 2005. Plant regrowth from potato shoot tips cryopreserved by a combined vitrification-droplet method. CryoLetters 26: 313-322.

Harding, K. 1997. Stability of the ribosomal RNA genes in Solanum tuberosum L. plants recovered from cryopreservation. CryoLetters 18: 217-230.

Harding, K. 2004. Genetic integrity of cryopreserved plant cells: A review. CryoLetters 25: 3-22.

Harding, K., Benson, E.E. \& Smith, H. 1991. The effects of pre-freeze in vitro culture period on the recovery of cryopreserved shoot tips of Solanum tuberosum. CryoLetters 12: 17-22.
Harding, K. \& Benson, E.E. 1994. A study of growth, flowering, and tuberisation in plants derived from cryopreserved potato shoot tips: implications for in vitro germplasm collections. CryoLetters 15: 59-66.

Harding, K., Johnston, J.W. \& Benson, E.E. 2008. Concepts in cryobionomics: A case study of Ribes genotype responses to cryopreservation in relation to thermal analysis oxidative stress nucleic acid methylation and transcriptional activity. In: Laamanen, J., Uosukainen, M., Häggman, H., Nukari, A., Rantala, S. (eds.). Cryopreservation of Crop Species in Europe. Proceedings of CRYOPLANET COST Action 871, $20^{\text {th }}-23^{\text {rd }}$ February 2008. Oulu, Finland. MTT Agrifood Research, Finland, Agrifood Research Working Papers 153. p.10-11.

Hirai, D. \& Sakai, A. 1999. Cryopreservation in in vitrogrown meristems of potato (Solanum tuberosum L.) by encapsulation-vitrification. Potato Research 42, 153-160.

Hoffman, N.E. \& Bischoff, J.C. 2004. Mechanisms of injury caused by in vivo freezing. In: Fuller, B. Lane, N. \& Benson, E.E. (eds.). Life in the Frozen State. CRC Press. Florida. p. 455-482.

Johnston, J.W., Harding, K., Bremner, D.H., Souch, G., Green, J., Lynch, P.T., Grout, B. \& Benson, E.E. 2005. HPLC analysis of plant DNA methylation: a study of critical methodological factors. Plant Physiology \& Biochemistry 43: 844-853.

Johnston, J.W., Harding, K. \& Benson, E.E. 2007. Antioxidant status and genotypic tolerance of Ribes in vitro cultures to cryopreservation. Plant Science 172: 524-534.

Johnston, J.W., Benson, E.E. \& Harding, K. 2009. Cryopreservation induces temporal DNA methylation epigenetic changes and differential transcriptional activity in Ribes germplasm. Plant Physiology \& Biochemistry 47: 123-131.

Keep, E., Grafton, W.H., Knoght, V.A. \& Cumming, I.G. 1983. The response of blackcurrant cultivars and selections to spring frosts. Journal of Horticultural Science 58: 535-540.

Keller, J.E.R. 2005. Improvement of cryopreservation results in garlic using low temperature preculture and highquality in vitro plantlets. CryoLetters 26: 357-366.

Keller, E.R.J. \& Dreiling, M. 2003. Potato cryopreservation in Germany - using droplet methods for the establishment of a new large collection. Acta Horticulturae (ISHS) 623: 193-200.

Keller, J.E.R., Kaczmarczyk, A. \& Senula, A. 2008. Cryopreservation for plant genebanks - a matter between high expectations and cautious reservation. CryoLetters 29: 53-62.

Khan, D.R., Ahmad, N., Anzar, M. \& Channa, A.A. 2009. Apoptosis in fresh and cryopreserved buffalo sperm. Theriogeneology 71: 872-876.

Kim, H-H., Yoon, J-W., Park, Y-E., Cho, E-G., Sohn, J-K., Kim, T-S. \& Engelmann, F. 2006. Cryopreservation of potato cultivated varieties and wild species: critical factors in droplet vitrification. CryoLetters 27: 223-234.

Kryszczuk, A., Keller, J., Grübe, M. \& Zimnoch-Guzowska, E. 2006. Cryopreservation of potato (Solanum tuberosum L.,) shoot tips using vitrification and droplet method. Journal of Food, Agriculture and Environment. 4: 196-200. 


\section{AGRICULTURAL AND FOOD SCIENCE}

\section{Harding, K. Exploring cryopreservation in clonally propagated crops}

Martinez, C.A., Loureiro, M.E., Oliva, M.A. \& Maestri, M. 2001. Differential responses of superoxide dismutase in freezing resistant Solanum curtilobum and freezing sensitive Solanum tuberosum subjected to oxidative and water stress. Plant Science 160: 505-515.

Mather, P.J.C., Modlibowska, I. \& Keep, E. 1980. Spring frost resistance in black currants Ribes nigrum L. Euphytica 29: 793-800.

Munné-Bosch, S. 2007. Aging in Perennials. Critical Reviews in Plant Sciences 26: 123-138.

Panis, B. 2007. CRYOPLANET - COST action 871. Cited 04 May 2009. Updated 18 Oct 2007. Available on the Internet: http://www.biw.kuleuven.be/dtp/tro/cost871/ home.htm.

Panis, B., Piette, B. \& Swennen, R. 2005. Droplet vitrification of apical meristems: a cryopreservation protocol applicable to all Musaceae. Plant Science 168: 45-55.

Reape, T.J. \& McCabe, P.F. 2008. Apoptotic-like programmed cell death in plants. New Phytologist 180: 13-26.

Reape, T.J. Molony, E.M. \& McCabe, P.F. 2008. Programmed cell death in plants: distinguishing between different modes. Journal of Experimental Botany 59: 435-44.

Reed, B.M. 2008. Cryopreservation - practical considerations. In: Reed, B. M. (ed.). Plant Cryopreservation: A Practical Guide. Chapter 1. Humana Press, $2^{\text {nd }}$ Edition. Springer, New York. p. 3-13.

Reed, B.M., Dumet, D.J., Denoma, J.M. \& Benson, E.E. 2001. Validation of cryopreservation protocols for plant germplasm conservation: a pilot study using Ribes $L$. Biodiversity and Conservation 10: 939-949.

Reed, B.M., Kovalchuk, I., Kushnarenko, S., Meier-Dinkel, A., Schoenweiss, K., Pluta, S., Straczynska, K. \& Benson, E.E. 2004. Evaluation of critical points in technology transfer of cryopreservation protocols to international plant conservation laboratories. CryoLetters 25: 341-352.

Reed, B.M., Schumacher, L., Dumet, D. \& Benson, E.E. 2005. Evaluation of a modified encapsulation-dehydration cryopreservation procedure incorporating sucrose pretreatments for the conservation of Ribes germplasm. In Vitro Cellular \& Developmental Biology Plant 41: 431-436.

Sakai, A. \& Engelmann, F. 2007. Vitrification, encapsulation-vitrification and droplet-vitrification: a review. CryoLetters 28: 151-172.

Schäfer-Menuhr, A., Schumacher, H-M. \& Mix-Wagner, G. 1997. Long-term storage of old potato varieties by cryopreservation of shoot tips in liquid nitrogen. Plant $\mathrm{Ge}$ netic Resources Newsletter 111: 19-24.

Sultan, S.E. 2000. Phenotypic plasticity for plant development, function and life history. Trends in Plant Science 5: 1360-1385.

Yoon, Ju-W., Kim, H-H., Ko, H-C., Hwang, H-S., Hong, E-S., Cho, E-G. \& Engelmann, F. 2006. Cryopreservation of cultivated and wild potato varieties by droplet vitrification: effect of subculture of mother-plants and of preculture of shoot tips. CryoLetter 27: 211-222. 